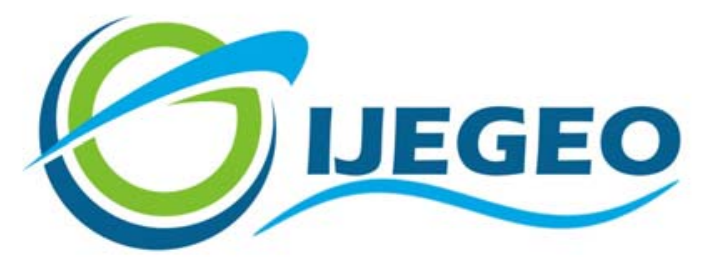

International Journal of Environment and Geoinformatics (IJEGEO) is an international, multidisciplinary, peer reviewed, open access journal.

\title{
Source identification of Polycyclic Aromatic Hydrocarbons (PAHs) in the urban environment of İstanbul
}

\author{
Günay Kural, Nuray Çağlar Balkıs and Abdullah Aksu
}

\author{
Editors \\ Prof. Dr. Cem Gazioğlu, Prof. Dr. Dursun Zafer Şeker, Prof. Dr. Ayşegül Tanık, \\ Prof. Dr. Şinasi Kaya, Assist. Prof. Dr. Volkan Demir
}

\section{Scientific Committee (2018)}

Assist. Prof. Dr. Abdullah Aksu, Prof. Dr. Bedri Alpar, Prof. Dr. Gülşen Altuğ, Prof. Dr. Lale Balas, Prof. Dr. Can Balas, Prof. Dr. Levent Bat, Prof. Dr. Bülent Bayram, Prof. Dr. Nuray Çağlar, Prof. Dr. Jadunandan Dash, Prof. Dr. A. Evren Erginal, Assoc. Prof. Dr. Ali Ertürk, Dr. Dieter Fritsch, Dr. Amin Gharehbaghi, Assoc. Prof. Dr. Tolga Görüm, Prof. Dr. Melike Gürel, Dr. Hakan Kaya , Prof. Dr. Fatmagül Kılıç, Assoc. Prof. Dr. Maged Marghany, Prof. Dr. Nebiye Musaoğlu, Prof. Dr. Masafumi Nakagawa, Prof. Dr. Haluk Özener, Prof. Dr. Erol Sarı, Prof. Dr. Elif Sertel, Prof. Dr. Nüket Sivri, Assoc. Prof. Dr. Füsun Balık Şanlı, Prof. Dr. Uğur Şanlı, Assoc. Prof. Dr. Hasan Özdemir, Prof. Dr. Taşkın Kavzoğlu Assoc. Prof. Dr. Oral Yağc1, Prof. Dr. Seyfettin Taş, Assoc. Prof. Dr. Ömer Suat Taşkın, Assoc. Prof. Dr. İ. Noyan Yılmaz, Assist. Prof. Dr. Baki Yokeş, Assit. Prof. Dr. Sibel Zeki 


\title{
Source identification of Polycyclic Aromatic Hydrocarbons (PAHs) in the urban environment of İstanbul
}

\author{
Günay Kural, Nuray Çağlar Balkı*, Abdullah Aksu \\ Istanbul University, Institute of Marine Science and Management, Department of Chemical Oceanography, Vefa 34134-00, \\ Istanbul, TURKEY \\ Corresponding author* \\ Received 20 Dec 2017 \\ Tel: $\quad+902124400000 / 26099$ \\ Accepted 01 Feb 2018 \\ E-mail :nbal@istanbul.edu.tr
}

\begin{abstract}
In this study, İstanbul-Tuzla Region atmosphere is selected as the working area because of this region contains shipyards and shipyard-related side product industries. To examine the PAHs as atmospheric inputs, 46 ambient air samples were collected in Tuzla region of Istanbul, Turkey at 2010. The sample collection was carried out as monthly. Additionally, the volume of the air was drawn and meteorological data recorded. The PAH concentrations were classified as hot and cold season samples. Due to the particle-bond character of PAHs and condensation in cold, the high concentrations were encountered in winter months. Monthly average total PAH concentrations fluctuated between $7.4 \mathrm{ng} \mathrm{m}-3$ (in March)- $0.05 \mathrm{ng} \mathrm{m}-3$ (in August). The concentrations of total PAHs in cold season were almost hundred times higher than those in hot season. The fossil fuel (gasoline and diesel fuel) combustion was found to be the main source of PAHs.
\end{abstract}

Keywords: Atmospheric pollution, polycyclic aromatic hydrocarbon (PAH), aerosol, İstanbul.

\section{Introduction}

Air pollution has emerged as a major health, environmental, economic and social problem all over the world (Aslan and Akyürek, 2018). During the past few decades, the atmosphere has been subjected to a large amount of contaminants via anthropogenic pollutants produced by both stationary (power plants, industrial and residential heating) and diffuse sources (road traffic) (Azimi et al. 2005). PAHs are toxic and carcinogenic and also mutagenic compounds that mainly result from the pyrolysis processes especially during the incomplete combustion of fossil fuels and biomass via the industrial processes, exhaust gases of the motor vessels and residential heating (Mastral and Callen 2000; Esen et al. 2008; Liu W. X. et al. 2009; Sisovic et al. 2008; Burak, et al., 2009). For environmental protection $16 \mathrm{PAH}$ compounds are defined as priority pollutants by US EPA (EPA, 2003). The names of the PAHs examined in this study are acenaphthene (Acp), acenaphtylene (AcPy), anthracene (Ant), benzo(a)anthracene $(\mathrm{BaA})$, benzo(a)pyrene $(\mathrm{BaP})$, benzo(b)flouranthene (BbFL), benzo(e)pyrene (BeP), benzo(k)flouranthene (BkFL), crysene (CHR), cyclopenta(cd)pyrene (CPcdP), flouranthene (FL), flourene (Flu), naphthalene (Nap), phenanthrene (PA), pyrene (Pyr).

PAHs are assumed to be Persistent Organic Pollutants - POPs (Bull, K. 2003, Liu Y. et al., 2006). PAHs have two or more benzonoid groups and no other elements except carbon and hydrogen (Henner et al. 1997). By the help of some chemical reactions such as sulfonation, nitration and photoaxidation they transform to more toxic compounds. When PAHs are released from the source they can be associated with particles by nucleation and condensation, forming particulate matter (PM) (Chester 1990). Two or three benzene rings existed in the vapor phase, while PAHs with more than five rings were observed primarily in the in the particulate form (Sisovic et al. 2008). 
In this study, for the first time particularly for this region, firstly it is aimed to determine the seasonal change in PAHs concentrations and secondly to find their sources and anthropogenic contributions. These results are a basic source for future studies and the study will be unique and a useful model for other industrial areas to determine pollution in a large range entirely on account of having examined organic pollutants.

\section{Materials And Methods Sampling procedure}

The sampling site is a residential area located in Turkey/İstanbul, TUDEV-Piri Reis University Campus (400 49' 05 66' $\mathrm{N}$ and 29020 '54 00' $\mathrm{E}$ ) at an altitude of $22 \mathrm{~m}$. The sampling point is $0.81 \mathrm{~km}$ away from the Marmara Sea. A High Volume Air Sampler (HVAS) was fixed at the top of the University Building roof to carry out the aerosols sampling by using Whatman filter papers (glass fiber) having a pore size of 0.1 $\mu \mathrm{m}$ were dried for 48 hours in a desiccator and weighed before and after the sampling. Then the filter papers were divided into two parts; each of the halves were wrapped in aluminum foil separately for PAHs analyses.

The sample collection was carried out at 2010. Five samples were taken for February, March, April, May, June, July, August, September and October. Only one sample was obtained for December. The volume of the air was drawn and meteorological data recorded.

\section{Chemical analysis Extraction}

Halves of the filter papers were cut into small pieces and then were extracted ultrasonically approximately for half an hour with $50 \mathrm{ml}$ of hexane-dichloromethane $(1: 1$, $\mathrm{v} / \mathrm{v})$ mixture for the analyses of the PAHs that become bonded with particles. Then the filtrates were evaporated (Sarrazine et al. 2007).

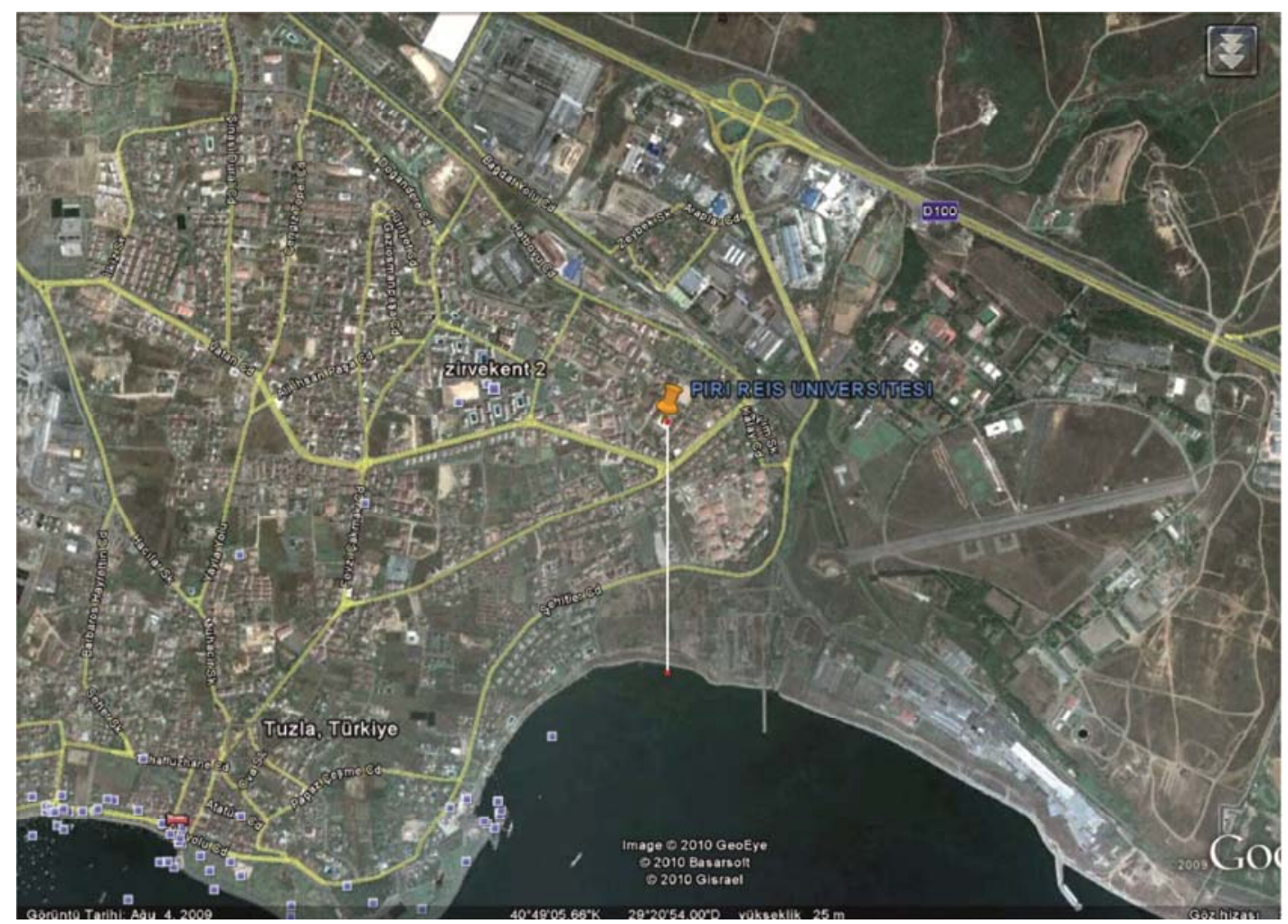

Fig. 1. Location of sampling area (Google Earth 2017) 
Table 1 Hot season monthly average concentrations of PAHs (ng m-3) in aerosols in 2010

\begin{tabular}{|c|c|c|c|c|c|c|c|c|c|c|c|c|c|c|c|c|c|c|c|c|c|}
\hline \multirow[t]{2}{*}{ PAHs } & \multicolumn{3}{|c|}{ May } & \multicolumn{3}{|c|}{ June } & \multicolumn{3}{|c|}{ July } & \multicolumn{3}{|c|}{ August } & \multicolumn{3}{|c|}{ September } & \multicolumn{3}{|c|}{ October } & \multicolumn{3}{|c|}{ Ave. of Hot Season } \\
\hline & Min & Max & $\mathrm{x}$ & Min & Max & $\mathrm{x}$ & Min & Max & $\mathrm{x}$ & Min & Max & $\mathrm{x}$ & Min & Max & $\mathrm{x}$ & Min & $\operatorname{Max}$ & $\bar{x}$ & Min & Max & $\mathrm{x}$ \\
\hline Nap & n.d. & 3.30 & 0.68 & n.d. & 2.28 & 0.54 & n.d. & n.d. & - & n.d. & n.d. & - & n.d. & n.d. & - & n.d. & n.d. & - & n.d. & 3.30 & 0.20 \\
\hline AcPy & n.d. & 0.04 & 0.01 & n.d. & 0.06 & 0.01 & n.d. & n.d. & - & n.d. & n.d. & - & n.d. & n.d. & - & n.d. & n.d. & - & n.d. & 0.04 & 0.003 \\
\hline Acp & n.d. & 0.05 & 0.01 & n.d. & 0.03 & 0.01 & n.d. & n.d. & - & n.d. & n.d. & - & n.d. & n.d. & - & n.d. & n.d. & - & n.d. & 0.05 & 0.003 \\
\hline Flu & n.d. & 0.02 & 0.01 & n.d. & 0.06 & 0.02 & n.d. & n.d. & - & n.d. & n.d. & - & n.d. & n.d. & - & n.d. & n.d. & - & n.d. & 0.06 & 0.005 \\
\hline PA & n.d. & 0.01 & 0.002 & n.d. & 0.06 & 0.01 & 0.06 & 0.017 & 0.004 & n.d. & n.d. & - & n.d. & 0.01 & 0.005 & n.d. & 0.08 & 0.02 & n.d. & 0.08 & 0.007 \\
\hline Ant & n.d. & n.d. & - & n.d. & n.d. & - & n.d. & n.d. & - & n.d. & n.d. & - & n.d. & n.d. & - & n.d. & n.d. & - & n.d & n.d & - \\
\hline FL & n.d. & 0.06 & 0.02 & n.d. & 0.07 & 0.02 & 0.006 & 0.02 & 0.001 & n.d. & 0.006 & 0.001 & 0.008 & 0.02 & 0.01 & 0.01 & 0.08 & 0.04 & n.d & 0.08 & 0.02 \\
\hline Pyr & 0.01 & 0.13 & 0.07 & n.d. & 0.35 & 0.10 & n.d. & 0.01 & 0.002 & n.d. & 0.002 & 0.0004 & 0.005 & 0.04 & 0.01 & 0.01 & 0.06 & 0.03 & n.d. & 0.26 & 0.07 \\
\hline CPcdP & 0.03 & 0.11 & 0.07 & 0.04 & 0.26 & 0.13 & n.d. & n.d. & - & n.d. & n.d. & - & n.d. & n.d. & - & 0.008 & 0.12 & 0.05 & n.d. & 0.13 & 0.04 \\
\hline $\mathrm{BaA}$ & n.d. & 0.01 & 0.002 & n.d. & 0.10 & 0.02 & n.d. & 0.006 & 0.001 & n.d. & 0.01 & 0.002 & n.d. & 0.04 & 0.01 & 0.02 & 0.15 & 0.07 & n.d & 0.15 & 0.003 \\
\hline CHR & n.d. & 0.01 & 0.002 & n.d. & n.d. & - & 0.002 & 0.16 & 0.14 & n.d. & n.d. & - & n.d. & 1.3 & 0.26 & n.d. & 0.004 & 0.0008 & n.d & 1.3 & 0.07 \\
\hline $\mathrm{BeP}$ & 0.01 & 0.34 & 0.13 & n.d. & 0.56 & 0.16 & 0.01 & 0.16 & 0.03 & 0.01 & 0.02 & 0.02 & n.d. & 0.02 & 0.008 & n.d. & 0.02 & 0.01 & n.d & 0.56 & 0.38 \\
\hline $\mathrm{BbFL}$ & n.d. & 0.14 & 0.06 & n.d. & 0.27 & 0.10 & 0.01 & 0.14 & 0.03 & 0.01 & 0.03 & 0.02 & n.d. & 0.03 & 0.02 & 0.007 & 0.09 & 0.05 & n.d & 0.14 & 0.05 \\
\hline $\mathrm{BkFL}$ & n.d. & 0.02 & 0.01 & n.d. & 0.45 & 0.14 & n.d. & 0.06 & 0.014 & n.d. & n.d. & - & n.d. & n.d. & - & n.d. & 0.07 & 0.015 & n.d. & 0.45 & 0.08 \\
\hline $\mathrm{BaP}$ & n.d. & 0.03 & 0.01 & n.d. & 0.05 & 0.02 & 0.003 & 0.09 & 0.02 & 0.03 & 0.08 & 0.01 & n.d. & 0.03 & 0.01 & n.d. & 0.02 & 0.1 & n.d & 0.08 & 0.03 \\
\hline Total & 0.05 & 4.27 & 1.07 & 0.04 & 4.6 & 1.27 & 0.09 & 0.66 & 0.24 & 0.05 & 0.15 & 0.05 & 0.013 & 1.49 & 0.33 & 0.06 & 0.7 & 0.3 & 0.05 & 4.27 & 0.54 \\
\hline
\end{tabular}




\section{Clean-up}

After holding the florisil at $1300 \mathrm{C}$ in 14 hours, it was deactivated with $3 \%$ of distilled water. The clean-up glass chromatographic column was prepared by using glass wool at the tip, and then the florisil was packed as an adsorbent and finally topped with anhydrous sodium sulfate. Conditioning carried out with $5 \mathrm{ml}$ hexane and $1 \mathrm{ml}$ sample of PAH extract was passed through the column. Then PAHs eluted with $5 \mathrm{ml}$ of hexzane:DCM $(1: 1, v / v)$ mixture. The eluate diluted to Gas Chromatography-Mass Spectrophotometer (GC-MS) to analysis (EPA 3620B).

In this study, except one of the PAH compound (IND) from sixteen $\mathrm{PAH}$ compounds, the other fifteen $\mathrm{PAH}$ compounds were analyzed. Recoveries of surrogate standards were 93-100 \% for PAHs analyses in this study (Table 1).

\section{Results And Discussions}

\section{PAH levels in aerosols}

The total PAH concentrations as the summation of 15 PAHs outstanding from the list of US-EPA are shown in Figure 2. It is seen that the concentration levels are high in cold months and were lower at hot months. The similar results are consistent many other researches (Wai Fon et al. 2007; Esen et al. 2008; Sisovic et al. 2008; Sharma et al. 2007). For this reason Table 2 and 3 data are grouped in order to determine the hot and cold season's monthly average concentrations of PAHs and their minimum and maximum levels in aerosol. While the hot season average PAHs concentration is $0.54 \mathrm{ng} \mathrm{m}-3$, the cold season average PAH concentration is $4.38 \mathrm{ng} \mathrm{m}-3$. The highest individual PAH concentration was measured for pyrene at March as $6.73 \mathrm{ng} \mathrm{m}-3$. BaP was found in high concentration only one time at February as $4.48 \mathrm{ng} \mathrm{m}-3$. The BeP was found in high concentrations all over the year with the highest value of $4.32 \mathrm{ng} \mathrm{m}-3$. The higher concentrations in cold season depend on to use of fossil fuel combustion for residential heating (Esen et al. 2008; Alp and Hanedar 2007; Sharma et al. 2007; Sisovic et al. 2008).

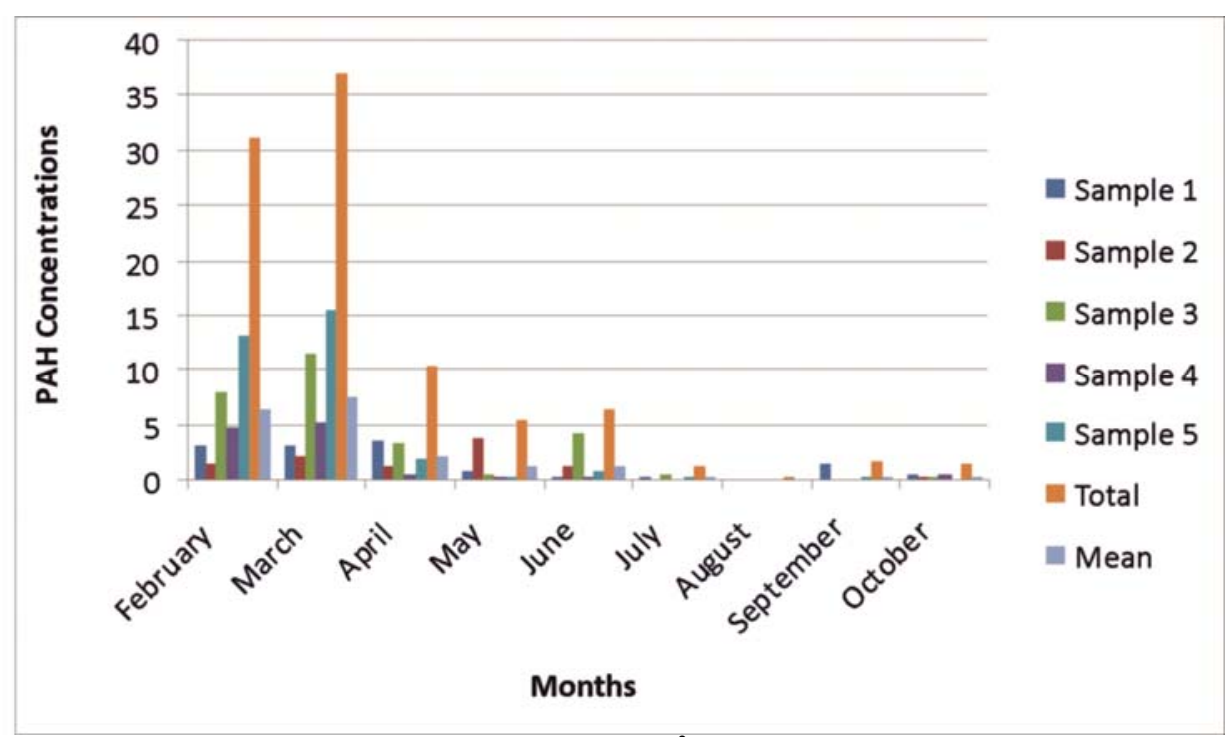

Fig. 2. Monthly total PAH concentrations $\left(\mathrm{ng} \mathrm{m}^{-3}\right)$.

In this study the frequency of high concentration of PAHs were found in 4-5 rings compounds. The most abundant PAHs shown in Fig. 3 are benzo(e)pyrene, benzo(b)flouranthene, benzo(k)flouranthene, cyclopenta(cd)pyrene and pyrene. Exceptionally, two ring compound of naphthalene were found in high concentrations. 
Table 2 Cold season monthly average concentrations of PAHs $\left(\mathrm{ng} \mathrm{m}^{-3}\right)$ in aerosols in 2010.

\begin{tabular}{|c|c|c|c|c|c|c|c|c|c|c|c|c|c|c|c|}
\hline \multirow[t]{2}{*}{ PAHs } & \multicolumn{3}{|c|}{ February } & \multicolumn{3}{|c|}{ March } & \multicolumn{3}{|c|}{ April } & \multicolumn{3}{|c|}{ December } & \multicolumn{3}{|c|}{ Ave. of Cold Season } \\
\hline & Min & Max & $\mathrm{x}$ & Min & Max & $\mathrm{x}$ & Min & Max & $\mathrm{x}$ & Min & Max & $\bar{x}$ & Min & $\operatorname{Max}$ & $\mathrm{x}$ \\
\hline Nap & n.d. & 0.30 & 0.07 & n.d. & 1.32 & 0.30 & n.d. & 0.12 & 0.04 & n.d. & - & - & n.d. & 1.32 & 0.10 \\
\hline AcPy & n.d. & 0.05 & 0.01 & n.d. & 0.08 & 0.03 & n.d. & 0.05 & 0.01 & n.d. & - & - & n.d. & 0.08 & 0.01 \\
\hline Acp & n.d. & 0.05 & 0.01 & n.d. & 0.11 & 0.04 & n.d. & 0.17 & 0.09 & n.d. & - & - & n.d. & 0.17 & 0.01 \\
\hline Flu & n.d. & 0.05 & 0.02 & n.d. & n.d. & - & n.d. & 0.05 & 0.02 & n.d. & - & - & n.d. & 0.05 & 0.01 \\
\hline PA & n.d. & 0.06 & 0.02 & n.d. & n.d. & - & n.d. & 0.02 & 0.004 & 0.04 & 0.04 & 0.04 & n.d. & 0.06 & 0.02 \\
\hline Ant & n.d. & 0.02 & 0.004 & n.d. & 0.01 & 0.002 & n.d. & 0.12 & 0.03 & n.d. & - & - & n.d. & 0.03 & 0.002 \\
\hline FL & n.d. & 0.29 & 0.11 & n.d. & 2.54 & 0.69 & n.d. & 0.20 & 0.04 & 0.15 & 0.15 & 0.15 & n.d. & 2.54 & 0.25 \\
\hline Pyr & n.d. & n.d. & - & 0.97 & 6.73 & 2.13 & 0.07 & 0.46 & 0.46 & 0.09 & 0.09 & 0.09 & n.d. & 6.73 & 0.67 \\
\hline CPcdP & 0.89 & 2.04 & 1.60 & 0.08 & 0.83 & 0.36 & 0.04 & 0.21 & 0.16 & 0.03 & 0.03 & 0.03 & 0.03 & 2.04 & 0.54 \\
\hline $\mathrm{BaA}$ & 0.02 & 1.70 & 0.49 & n.d. & 0.11 & 0.04 & n.d. & 0.05 & 0.01 & 0.07 & 0.07 & 0.07 & n.d. & 1.70 & 0.15 \\
\hline CHR & n.d. & 0.20 & 0.07 & n.d. & 0.74 & 0.23 & n.d. & 0.03 & 0.01 & 1 & 1 & 1 & n.d & 1 & 0.33 \\
\hline $\mathrm{BeP}$ & n.d. & 2.23 & 0.99 & 0.60 & 4.32 & 1.62 & n.d. & 1.46 & 0.61 & 0.02 & 0.02 & 0.02 & n.d & 1.62 & 0.81 \\
\hline $\mathrm{BbFL}$ & n.d. & 1.71 & 0.60 & 0.01 & 2.07 & 0.71 & 0.02 & 0.67 & 0.28 & 0.03 & 0.03 & 0.03 & n.d & 2.07 & 0.41 \\
\hline $\mathrm{BkFL}$ & n.d. & 3.78 & 1.16 & 0.30 & 1.52 & 0.95 & n.d. & 0.55 & 0.19 & n.d. & - & - & n.d & 3.78 & 0.58 \\
\hline $\mathrm{BaP}$ & 0.03 & 4.48 & 1.06 & n.d. & 0.97 & 0.31 & 0.01 & 0.09 & 0.09 & 0.003 & 0.003 & 0.003 & n.d & 4.48 & 0.37 \\
\hline Total & 0.94 & 14.92 & 6.22 & 1.96 & 21.35 & 7.4 & 0.23 & 4.25 & 2.05 & 1.4 & 1.4 & 1.4 & 0.23 & 21.35 & 4.38 \\
\hline \multicolumn{16}{|c|}{ Table 3 Seasonal BaP values } \\
\hline \multicolumn{3}{|c|}{ PAHs } & \multicolumn{3}{|c|}{$\begin{array}{c}\text { TEF values by } \\
\text { Nisbet and LaGoy }\end{array}$} & \multicolumn{3}{|c|}{$\begin{array}{c}\text { Hot season } \\
\text { PAHs }\left(\text { ng m }^{-3}\right)\end{array}$} & \multicolumn{2}{|c|}{$\begin{array}{c}\text { Hot season TEF } \\
\text { values }\end{array}$} & \multicolumn{3}{|c|}{$\begin{array}{c}\text { Cold season } \\
\text { PAHs }\left(\text { ng m }^{-3}\right)\end{array}$} & \multicolumn{2}{|c|}{$\begin{array}{l}\text { Cold season } \\
\text { TEF values }\end{array}$} \\
\hline \multicolumn{3}{|c|}{ Nap } & \multicolumn{3}{|c|}{0.001} & \multicolumn{2}{|c|}{0.20} & & \multicolumn{2}{|c|}{0.0002} & & \multicolumn{2}{|c|}{0.10} & \multicolumn{2}{|c|}{0.0001} \\
\hline \multicolumn{3}{|c|}{ AcPy } & \multicolumn{3}{|c|}{0.001} & \multicolumn{2}{|c|}{0.003} & & \multicolumn{2}{|c|}{0.000003} & & 0.01 & & & 0.00001 \\
\hline & Acp & & & 001 & & & 03 & & & 0003 & & 0.01 & & & 0.00001 \\
\hline & Flu & & & 001 & & & 05 & & & 0005 & & 0.01 & & & 0.00001 \\
\hline & PA & & & 001 & & & 07 & & & 007 & & 0.02 & & & 0.00002 \\
\hline & Ant & & & 01 & & & s. & & & - & & 0.00 & & & 0.00002 \\
\hline & FL & & & 001 & & & 22 & & & 002 & & 0.25 & & & .000025 \\
\hline & Pyr & & & 001 & & & 97 & & & 007 & & 0.67 & & & .000067 \\
\hline & PedP & & & - & & & 34 & & & - & & 0.54 & & & - \\
\hline & $\mathrm{BaA}$ & & & .1 & & & 03 & & & 003 & & 0.15 & & & 0.015 \\
\hline & CHR & & & 01 & & & 77 & & & 007 & & 0.33 & & & 0.0033 \\
\hline & $\mathrm{BeP}$ & & & - & & & 38 & & & - & & 0.81 & & & - \\
\hline & $\mathrm{BbFL}$ & & & .1 & & & 55 & & & 05 & & 0.41 & & & 0.041 \\
\hline & $\mathrm{BkFL}$ & & & 1 & & & 08 & & & 08 & & 0.58 & & & 0.058 \\
\hline & $\mathrm{BaP}$ & & & 1 & & & 93 & & & 03 & & 0.37 & & & 0.37 \\
\hline & Total & & & & & & 54 & & & 04 & & 4.38 & & & 0.49 \\
\hline
\end{tabular}




\section{Number of Days}

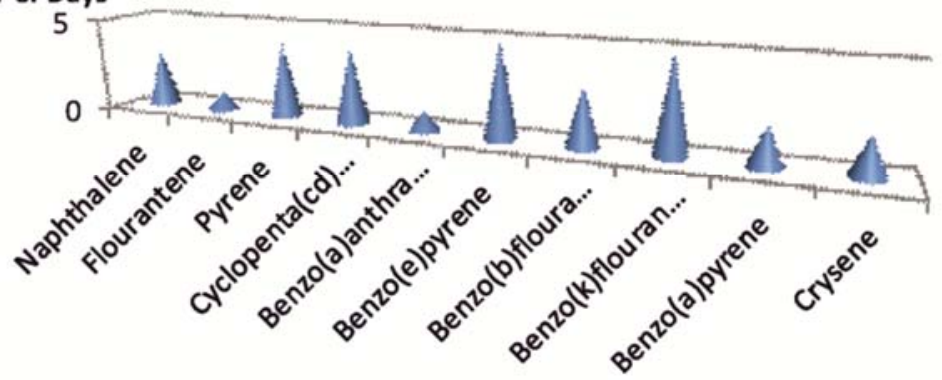

- PAHs

Fig. 3. Frequency of high concentration PAHs.

The high correlations were observed generally at higher ring compounds; such as (CPcdP) and BaP $(\mathrm{r}=+0.99), \mathrm{BeP}$ and $\mathrm{BbFL}$ $(\mathrm{r}=+0.98), \mathrm{BbFL}$ and $\mathrm{BkFL}(\mathrm{r}=+96), \mathrm{BaA}$ and $\mathrm{BaP}(\mathrm{r}=+96), \mathrm{FL}$ and $\mathrm{Pyr}(\mathrm{r}=+95)$, AcPy and Bep ( $\mathrm{r}=+90), \mathrm{BeP}$ and BkFL $(\mathrm{r}=$ $+90)$. The correlations between two or three ring compounds were found medium or lower level (Table 4). In general, the lower molecular PAHs (two or three rings) appeared to be associated with the vapor Table 4 Diagnosis ratios of aerosol PAHs phase, and high molecular weight PAHs (five or six rings) were primary attached with the particulate phase (Lui $G$ et al. 2010). For heavy PAHs, owing to the low volatility and close association with particles, once deposited from air, they may be expected to cycle in the soil-watersediment system, rather than "hoping" upward to a higher altitude under normal ambient temperatures (Liu X., et al. 2005).

\begin{tabular}{cccccccc}
\hline Molecular ratios & $\begin{array}{c}\text { This study } \\
\text { hot season }\end{array}$ & $\begin{array}{c}\text { This study } \\
\text { cold season }\end{array}$ & Gasoline & $\begin{array}{c}\text { Vehicular } \\
\text { emissions }\end{array}$ & Diesel & $\begin{array}{c}\text { Wood } \\
\text { combustion }\end{array}$ & $\begin{array}{c}\text { Used } \\
\text { motor oil }\end{array}$ \\
\hline $\mathrm{BaA} / \mathrm{CHR}$ & 0.04 & 0.45 & $0.28-1.20$ & $0.40-0.58$ & $0.17-0.36$ & 0.93 & 0.5 \\
$\mathrm{BeP} / \mathrm{BaP}$ & 12.67 & 2.20 & $1.1-1.3$ & - & $2.0-2.5$ & 0.44 & - \\
$\mathrm{Pyr} / \mathrm{BaP}$ & 2.33 & 1.81 & 0.85 & - & 0.81 & 0.71 & - \\
$\mathrm{CHR} / \mathrm{BeP}$ & 0.18 & 0.41 & 2.50 & - & 1.60 & 2.40 & - \\
\hline
\end{tabular}

$\mathrm{BaP}$ is the $\mathrm{PAH}$ most widely studied and the abundance of information on toxicity and occurrence of PAHs is related to this compound. The natural background level of $\mathrm{BaP}$ may be near to zero. The current annual mean concentration of $\mathrm{BaP}$ in major European areas is in the range of 1-10 ng m3. In rural areas, the concentrations are $<1$ ng m-3 (WHO 2000; Sisovic et al. 2008). Maximum concentration of $\mathrm{BaP}$ due to high carcinogenic property by WHO (1998) is 1 ng m-3. Table 5 shows the TEFs (Toxic Equivalency Factors as $\mathrm{BaP}$ ) values of 13 PAHs of the study that proposed before by Nisbet and LaGoy (1992). Hot and cold season TEFs values were found as $0.04 \mathrm{ng}$ $\mathrm{m}-3$ and $0.49 \mathrm{ng} \mathrm{m}-3$ respectively. Both seasonal concentrations are below the carcinogenic limit value. For the following two studies; BaP equivalency calculations were realized for 16 PAHs In the first one, Alp and Hanedar (2008) were found 2.648 ng $\mathrm{m}-3$ in a traffic congested area. In the second one, Chantara and Sangchan (2009) were found $0.99 \pm 0.31 \mathrm{ng} \mathrm{m}-3$ in a suburban area, $0.92 \pm 0.46 \mathrm{ng} \mathrm{m}-3$ in a community area, $1.70 \pm 0.55 \mathrm{ng} \mathrm{m}-3$ and $1.64 \pm 0,41$ as $\mathrm{ng} \mathrm{m}-3$ in two traffic congested area. The genotoxic risk levels were high at the industrialized urban localities, when the pollution level of traffic emissions and emissions from industry and household heating were high (Skarek et al. 2007). 
Table 5 Comparison of mean concentrations $\left(\mathrm{ng} \mathrm{m}^{-3}\right)$ of total PAHs with other studies

\begin{tabular}{|c|c|c|c|c|}
\hline $\begin{array}{l}\text { Total } \\
\text { PAHs } \\
\end{array}$ & Sampling Site & Mean $\left(\mathrm{ng} \mathrm{m}^{-3}\right)$ & Cities & References \\
\hline$\sum 15$ PAHs & $\begin{array}{l}\text { Residential area (near } \\
\text { industrial area) }\end{array}$ & 2.37 & $\begin{array}{l}\text { TR İstanbul. } \\
\text { Tuzla }\end{array}$ & This study \\
\hline$\sum 13$ PAHs & Near coal reserve & $0.051-1.791$ & Brazil. Candiota & Dallarosa et al. (2005) \\
\hline$\sum 13$ PAHs & Suburban area. traffic & 2.39 & Japan. Hiroshima & Wai Fon et al. (2007) \\
\hline$\sum 16$ PAHs & Urban area & $28.53-362.15$ & China. Beijing & $\begin{array}{l}\text { Bin Liu L, et al. } \\
(2007)\end{array}$ \\
\hline$\sum 15$ PAHs & Urban area & 86.0 & China. Nanjing & Wang. et al. (2006) \\
\hline$\sum 16$ PAHs & Traffic congestion & $84.63 \pm 46.66$ & $\begin{array}{l}\text { TR. İstanbul. } \\
\text { Göztepe. }\end{array}$ & $\begin{array}{l}\text { Alp and Hanedar } \\
(2008)\end{array}$ \\
\hline$\sum 16$ PAHs & $\begin{array}{l}\text { Local } \\
\text { micrometeorological area }\end{array}$ & 26.7 & Shenzen. China & Liu et al. (2010) \\
\hline $\begin{array}{l}\sum 16 \text { PAHs } \\
\text { (three } \\
\text { sites) }\end{array}$ & $\begin{array}{l}\text { Suburban area } \\
\text { Community area } \\
\text { Traffic congestion }\end{array}$ & $\begin{array}{l}2.7-8.4 \\
3.9-9.1 \\
7.6-16.6\end{array}$ & $\begin{array}{l}\text { Thailand. Chiang } \\
\text { Mai }\end{array}$ & $\begin{array}{l}\text { Chantara and } \\
\text { Sangchan (2009) }\end{array}$ \\
\hline
\end{tabular}

Relationship between PAH concentrations and meteorological conditions

Table 7 is prepared to observe the effect of meteorological conditions on PAHs concentrations in the ambient air of the region during the sampling periods. As it was mentioned in many studies that the main source of the PAHs in the atmosphere was the emissions of fossil fuel combustions for heating purposes and increase in traffic volume in winter (Liu X. et al. 2005; Liu G. et al. 2010; Liu Y. et al. 2006. Bin Liu L. et al. 2007, Dallarosa et al. 2005). But also the meteorological conditions cause to hold the produced PAHs in the region. Emissions from fossil fuel combustion cannot be easily dispersed depending on the high humidity and low temperature values (Sharma et al. 2007).

The good correlations had been found between meteorological parameters such as temperature, wind speed and direction with PAHs concentrations (Wai Fon et al. 2007; Fang et al. 2004; Panther, Hooper and Tapper 1999). In this study, the poor correlation was seen between temperature and total PAHs concentrations as $\mathrm{r}=+0.60$ (Fig. 4).

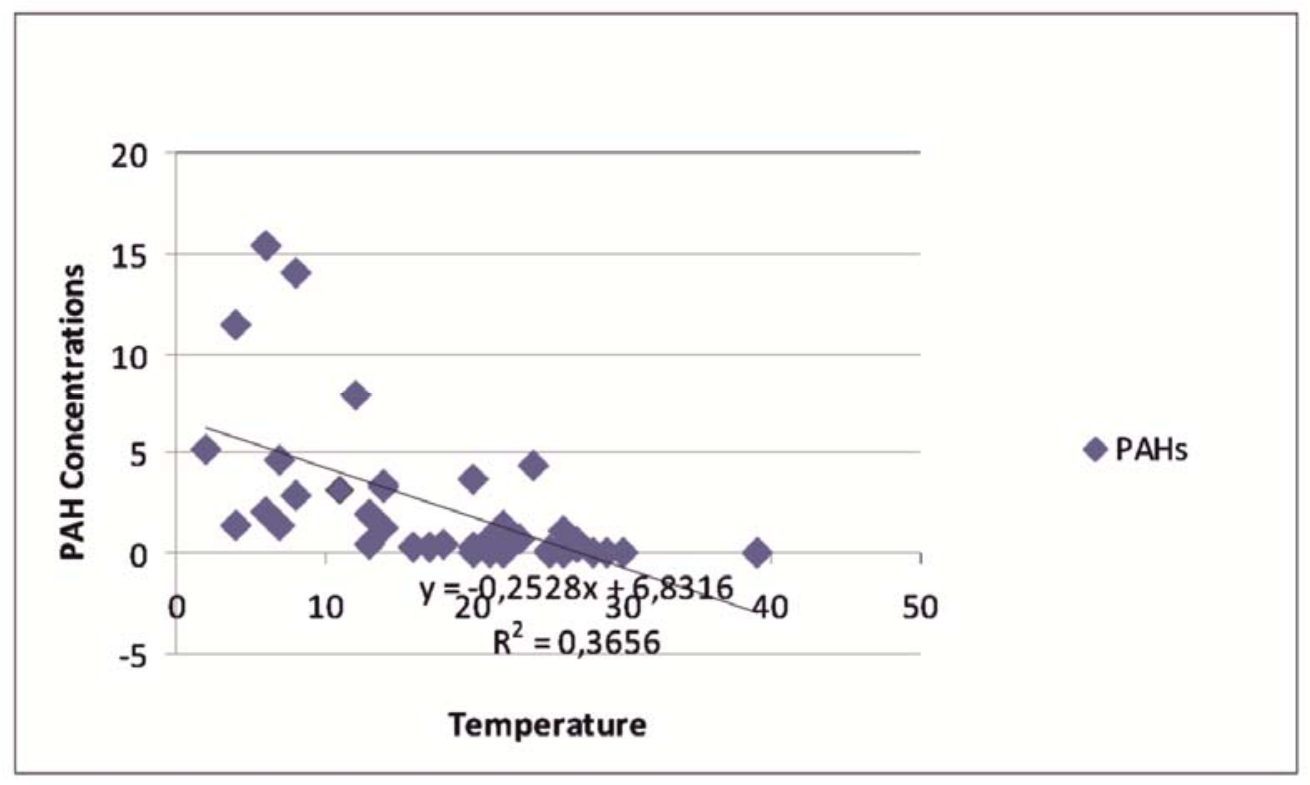

Fig. 4. Correlation of temperature $\left({ }^{0} \mathrm{C}\right)$ and total PAH concentrations $\left(\mathrm{ng} \mathrm{m}^{-3}\right)$. 
The weak correlation $(\mathrm{r}=+0.27)$ was seen between humidity and total PAH concentrations (Fig. 5). But the naphthalene as the smallest member of PAHs was found as four times in high concentrations (Fig. 3) even if it must be found in gaseous state in the atmosphere. The rainy weather or high relative humidity during or before sampling period caused to be found Nap in condensed phase as liquid form bounding particle in the region aerosol (Table 6). In February Sample No:3 also the dust concentration was in between $0.25-0.50 \mathrm{mg} \mathrm{m}-3$. The elevated dust concentration causes to increase the adsorption of naphthalene on particulate matter. A combination of particulate scavenging and cold condensation were proposed as a major mechanism for the compositional fractionation of PAHs along the altitudinal profile (Liu X. et al. 2005).

Table 6. Relation between meteorological parameters with Nap concentration

\begin{tabular}{|c|c|c|c|c|}
\hline $\begin{array}{l}\text { Sample } \\
\text { No. }\end{array}$ & $\begin{array}{c}\text { Nap } \\
\left(\mathrm{ng} / \mathrm{m}^{3}\right)\end{array}$ & Humidity(\%) & Precipitation(mm) & Temp. $\left({ }^{0} \mathrm{C}\right)$ \\
\hline Feb 3 & 3.30 & $\begin{array}{c}\text { After } 2 \text { high humidity } \\
\text { days }(80 \& 70)+\text { during sampling } \\
(70)\end{array}$ & $\begin{array}{c}\text { After } 2 \text { rainy days } \\
(0.2 \& 0.6)+\text { during sampling }(0.4)\end{array}$ & 12 \\
\hline March 4 & 1.32 & $\begin{array}{l}\text { 3days before up to } 84+\text { during } \\
\text { sampling (63) }\end{array}$ & $\begin{array}{l}\text { After } 1 \text { rainy day }(0.4) \text { and during } \\
\text { sampling }(0.2)\end{array}$ & 2 \\
\hline May 2 & 3.65 & $\begin{array}{c}\text { After } 1 \text { high humidity days }(85)+ \\
\text { during sampling }(60)\end{array}$ & After 1 rainy day (0.6) & 20 \\
\hline June 2 & 2.28 & $\begin{array}{l}1 \text { day ago (62) and sampling day } \\
\text { (70) }\end{array}$ & $\begin{array}{c}\text { After } 1 \text { rainy day }(8)+\text { during } \\
\text { sampling (8) }\end{array}$ & 26 \\
\hline
\end{tabular}

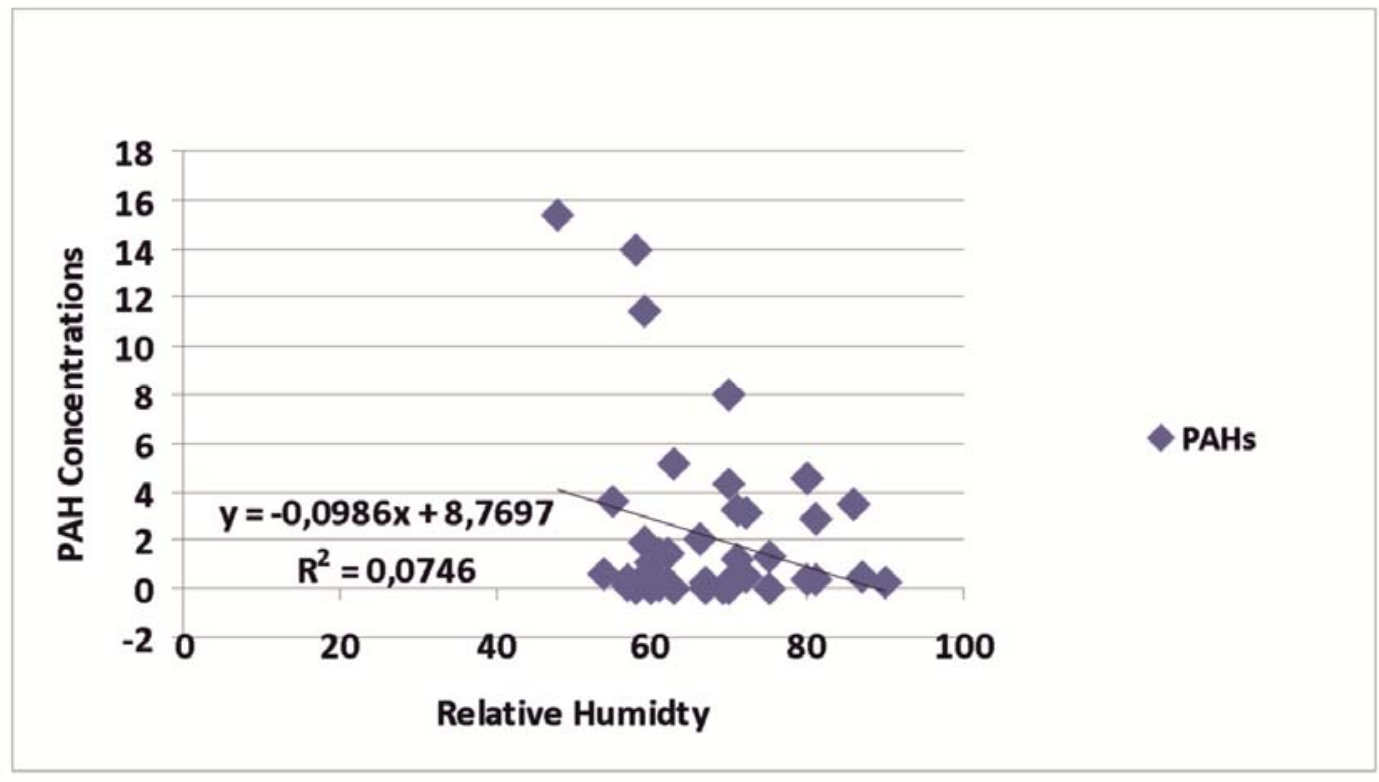

Fig. 5. Correlation of relative humidity (\%) and total PAH $\left(\mathrm{ng} \mathrm{m}^{-3}\right)$ 
Table 7. Meteorological parameters in the year 2010

\begin{tabular}{|c|c|c|c|c|c|c|}
\hline $\begin{array}{l}\text { Sampling } \\
\text { Date/ } \\
\text { Duration }\end{array}$ & No & $\begin{array}{l}\text { Average } \\
\text { Temp. } \\
\left({ }^{0} \mathrm{C}\right)\end{array}$ & $\begin{array}{c}\text { Wind-Speed } \\
\text { (Ave-Max) } \\
(\mathrm{km} / \mathrm{h})\end{array}$ & $\begin{array}{c}\text { Wind } \\
\text { Direction }\end{array}$ & $\begin{array}{l}\text { Precipitation } \\
(\mathrm{mm})\end{array}$ & $\begin{array}{c}\text { Relative } \\
\text { Humidity } \\
(\%) \\
\text { (Ave-Max) }\end{array}$ \\
\hline $12.02 .2010 /(10: 00)-13.02 .2010 /(09: 30)$ & 1 & 11 & $12-33$ & SW & 0.2 & $72-83$ \\
\hline $16.02 .2010 /(16: 00)-17.02 .2010 /(09: 00)$ & 2 & 7 & $7-15$ & $\mathrm{NE}$ & 0.6 & $61-70$ \\
\hline $17.02 .2010 /(17: 00)-18.02 .2010(09: 00)$ & 3 & 12 & $10-23$ & SW & 0.4 & $52-70$ \\
\hline $18.02 .2010 /(17: 00)-19.02 .2010 /(09: 30)$ & 4 & 7 & $8-20$ & SW & - & $80-90$ \\
\hline $22.02 .2010 /(17: 00)-23.02 .2010 /(09: 00)$ & 5 & 8 & $6-18$ & S-SW & - & $58-71$ \\
\hline $11.03 .2010 /(17: 00)-12.03 .2010 /(09: 00)$ & 1 & 8 & $16-28$ & N-SW & showery & $81-84$ \\
\hline $15.03 .2010 /(17: 00)-16.03 .2010 /(09: 00)$ & 2 & 6 & $11-20$ & $\mathrm{~N}-\mathrm{S}$ & - & $66-59$ \\
\hline $16.03 .2010 /(17: 00)-17.03 .2010 /(09: 00)$ & 3 & 4 & $15-32$ & $\mathrm{~S}$ & 0.4 & $59-63$ \\
\hline $17.03 .2010 /(17: 00)-18.03 .2010 /(12: 00)$ & 4 & 2 & $8-17$ & $\mathrm{~N}-\mathrm{S}$ & 0.2 & $48-63$ \\
\hline $18.03 .2010 /(17: 00)-19.03 .2010 /(09: 00)$ & 5 & 6 & $6-15$ & N-E & - & $48-51$ \\
\hline $15.04 .2010 /(18: 00)-16.04 .2010 /(10: 30)$ & 1 & 14 & $5-15$ & N-SW & - & $86-90$ \\
\hline $16.04 .2010 /(18: 00)-17.04 .2010 /(19: 00)$ & 2 & 14 & $20-32$ & $\mathrm{~N}$ & - & $71-57$ \\
\hline $19.04 .2010 /(09: 30)-20.04 .2010 /(09: 30)$ & 3 & 14 & $10-20$ & $\mathrm{~N}-\mathrm{S}$ & 0.6 & $71-100$ \\
\hline $20.04 .2010 /(17: 30)-21.04 .2010 /(17: 00)$ & 4 & 13 & $15-35$ & $\mathrm{~N}$ & 0.8 & $80-100$ \\
\hline $21.04 .2010 /(17: 00)-22.04 .2010 /(09: 30)$ & 5 & 13 & $11-22$ & W-SW & - & $59-100$ \\
\hline $12.05 .2010 /(17: 00)-13.05 .2010 /(09: 00)$ & 1 & 21 & $7-15$ & N-E & 0.6 & $60-85$ \\
\hline $14.05 .2010 /(17: 00)-15.05 .2010 /(17: 00)$ & 2 & 20 & $10-20$ & S-E & - & $55-60$ \\
\hline $17.05 .2010 /(17: 00)-18.05 .2010 /(17: 00)$ & 3 & 18 & $22-32$ & $\mathrm{~S}-\mathrm{N}$ & - & $60-70$ \\
\hline $18.05 .2010 /(17: 00)-19.05 .2010 /(16: 00)$ & 4 & 16 & $12-17$ & S-W & - & $57-70$ \\
\hline $20.05 .2010 /(16: 00)-21.05 .2010 /(13: 00)$ & 5 & 17 & $8-22$ & $\mathrm{~S}-\mathrm{N}$ & - & $40-57$ \\
\hline $18.06 .2010 /(17: 00)-19.06 .2010 /(18: 00)$ & 1 & 25 & $17-26$ & $\mathrm{~N}$ & 8 & $62-59$ \\
\hline $19.06 .2010 /(18: 00)-20.06 .2010 /(16: 00)$ & 2 & 26 & $9-20$ & $\mathrm{~N}-\mathrm{S}$ & 8 & $60-70$ \\
\hline $20.06 .2010 /(16: 00)-21.06 .2010 /(09: 00)$ & 3 & 24 & $10-20$ & $\mathrm{~N}-\mathrm{S}$ & - & $70-80$ \\
\hline $21.06 .2010 /(17: 00)-22.06 .2010 /(17: 00)$ & 4 & 22 & $13-39$ & $\mathrm{~S}$ & - & $54-57$ \\
\hline $22.06 .2010 /(17: 00)-23.06 .2010 /(17: 00)$ & 5 & 23 & $12-24$ & E-SW & - & $54-65$ \\
\hline $19.07 .2010 /(18: 00)-20.07 .2010 /(17: 00)$ & 1 & 26 & $15-37$ & $\mathrm{~N}$ & - & $62-89$ \\
\hline $20.07 .2010 /(17: 00)-21.07 .2010 /(17: 00)$ & 2 & 25 & $11-24$ & $\mathrm{~N}$ & - & $69-83$ \\
\hline $21.07 .2010 /(17: 00)-22.07 .201 /(18: 00)$ & 3 & 27 & $15-30$ & $\mathrm{~N}$ & - & $72-94$ \\
\hline $22.07 .2010 /(17: 00)-23.07 .2010 /(17: 00)$ & 4 & 29 & $15-26$ & $\mathrm{~N}$ & - & $63-89$ \\
\hline $23.07 .2010 /(17: 00)-24.07 .2010 /(09: 00)$ & 5 & 27 & $11-18$ & $\mathrm{~N}-\mathrm{S}$ & - & $67-90$ \\
\hline $09.08 .2010 /(17: 00)-10.08 .2010 /(11: 00)$ & 1 & 25 & $15-35$ & $\mathrm{~N}$ & exist & $75-89$ \\
\hline $10.08 .2010 /(17: 00)-11.08 .2010 /(17: 00)$ & 2 & 30 & $14-39$ & $\mathrm{~N}$ & - & $70-89$ \\
\hline $11.08 .2010 /(17: 00)-12.08 .2010 /(17: 00)$ & 3 & 28 & $14-28$ & $\mathrm{~N}$ & - & $67-83$ \\
\hline $12.08 .2010 /(17: 00)-13.08 .2010 /(17: 00)$ & 4 & 39 & $9-16$ & $\mathrm{~N}-\mathrm{S}$ & - & $69-94$ \\
\hline $13.08 .2010 /(17: 00)-14.08 .2010 /(11: 00)$ & 5 & 30 & $9-22$ & Variable & - & $69-50$ \\
\hline $20.09 .2010 /(17: 00)-21.09 .2010 /(17: 00)$ & 1 & 22 & $14-39$ & N-NW & - & $75-100$ \\
\hline $21.09 .2010 /(17: 00)-22.09 .2010 /(17: 00)$ & 2 & 20 & $27-41$ & N-NE & - & $70-88$ \\
\hline $22.09 .2010 /(17: 00)-23.09 .2010 /(17: 00)$ & 3 & 21 & $21-33$ & $\mathrm{~N}$ & - & $58-63$ \\
\hline $26.09 .2010 /(17: 00)-27.09 .2010 /(17: 00)$ & 4 & 26 & $8-20$ & S-SE & - & $60-78$ \\
\hline $28.09 .2010 /(17: 00)-29.09 .2010 /(17: 00)$ & 5 & 22 & $10-18$ & S-SW & - & $61-78$ \\
\hline $13.10 .2010 /(17: 00)-14.10 .2010 /(17: 30)$ & 1 & 17 & $7-15$ & $\mathrm{~N}-\mathrm{S}$ & 5 & $87-94$ \\
\hline $14.10 .2010 /(17: 00)-15.10 .2010 /(14: 00)$ & 2 & 17 & $13-18$ & NE & 9 & $90-100$ \\
\hline $16.10 .2010 /(19: 00)-17.10 .2010 /(19: 00)$ & 3 & 18 & $9-18$ & $\mathrm{~N}-\mathrm{NE}$ & 2 & $90-100$ \\
\hline $17.10 .2010 /(18: 00)-18.10 .2010 /(18: 00)$ & 4 & 18 & $5-13$ & S-SW & - & $81-94$ \\
\hline $18.10 .2010 /(18: 00)-19.10 .2010 /(19: 00)$ & 5 & 19 & $9-17$ & $\mathrm{~S}$ & 2 & $70-88$ \\
\hline $13.12 .2010 /(17: 00)-14.12 .2010 /(17: 30)$ & 1 & 4 & $15-26$ & $\mathrm{~N}$ & 8 & $62-87$ \\
\hline
\end{tabular}

BeP being the first, BbFL and BkFL are the PAHs mostly encountered in elevated concentrations in hot season and especially in cold season (Table 2 and 3 ). $\mathrm{Bi}$, et al. (2003) pointed out that the two and threering PAHs generally had a greater vapor phase component, while the higher molecular weight exhibited greater association with the particulate phase. Anthracene concentrations could not be detected in hot season; it was also measured only four times (Sample No: February 5, March 2, April 3 and 5) in cold season (Table 3). As seen from Table 7, the sampling day was rainy and there was a high relative humidity during sampling or the previous period. Two similar situations were observed that Ant concentration was higher in wet deposition rather than dry deposition (Pekey et al. 2007; Gaga and Tuncel 2003). 
Even if the use of natural gas for heating purposes in the region, the concentration of CHR was found to be at a high level twice; during September and December relating to the use of coal (Esen et al. 2008). Also, higher CHR concentrations were proposed relative to lower atmospheric temperature during winter season (Pekey et al. 2007; Fang* et al. 2004).

\section{PAH ratios for source identification}

In many studies molecular ratios are used to determine the source of the aerosol PAHs (Wai Fon et al. 2007; Fang et al. 2004; Bourotte et al. 2005; Khalli et al. $1995 \mathrm{Li}$ and Kamens 1993). Wai Fon et al. (2007) used $\mathrm{BaA} / \mathrm{CHR}, \mathrm{BeP} / \mathrm{BaP}, \mathrm{Pyr} / \mathrm{BaP}$ and $\mathrm{CHR} / \mathrm{BeP}$ ratios to detect the molecular diagnosis of aerosol PAHs, and obtained the results as $0.51, \quad 0.80, \quad 1.00$ and 1.95 respectively and determined the PAHs emission sources derived from gasoline. The same ratios were used in this study as hot and cold seasons (Table 8). In hot season, $\mathrm{Pyr} / \mathrm{BaP}$ and especially $\mathrm{BeP} / \mathrm{BaP}$ ratios are excessively high values since the concentrations of $\mathrm{BaP}$ levels are so low and BeP levels are relatively high (Table 2). The high concentrations of $\mathrm{BeP}$ also cause to move down the $\mathrm{CHR} / \mathrm{BeP}$ ratio. The source profiles of vehicle exhausts are heavily influenced by many factors, such as fuel quality and engine power status (Shen et al. 2010). It is clearly seen that in the cold season the ratio of $\mathrm{BeP} / \mathrm{BaP}$ point outs diesel emissions with the value of 2.20. The $\mathrm{BaA} / \mathrm{CHR}$ ratio takes place in the gasoline range with the value of 0.45 . It can be concluded that the PAHs in the region atmosphere is dominated by traffic emissions. These results also support the idea of condensation of emissions of PAHs at cold weather (Sharma et al. 2006).

Table 8. Diagnosis ratios of aerosol PAHs

\begin{tabular}{|c|c|c|c|c|c|c|c|c|}
\hline $\begin{array}{l}\text { Molecular } \\
\text { ratios }\end{array}$ & $\begin{array}{l}\text { This } \\
\text { study } \\
\text { hot } \\
\text { season }\end{array}$ & $\begin{array}{l}\text { This } \\
\text { study } \\
\text { cold } \\
\text { season }\end{array}$ & Gasoline & \multicolumn{2}{|c|}{$\begin{array}{l}\text { Vehicular } \\
\text { emissions }\end{array}$} & Diesel & $\begin{array}{c}\text { Wood } \\
\text { combustion }\end{array}$ & $\begin{array}{l}\text { Used } \\
\text { motor } \\
\text { oil }\end{array}$ \\
\hline $\mathrm{BaA} / \mathrm{CHR}$ & 0.04 & 0.45 & $\begin{array}{c}0.28- \\
1.20\end{array}$ & \multicolumn{2}{|c|}{$0.40-0.58$} & $\begin{array}{l}0.17- \\
0.36\end{array}$ & 0.93 & 0.5 \\
\hline $\mathrm{BeP} / \mathrm{BaP}$ & 12.67 & 2.20 & $1.1-1.3$ & \multicolumn{2}{|c|}{ - } & $2.0-2.5$ & 0.44 & - \\
\hline Pyr/BaP & 2.33 & 1.81 & 0.85 & \multicolumn{2}{|c|}{ - } & 0.81 & 0.71 & - \\
\hline $\mathrm{CHR} / \mathrm{BeP}$ & 0.18 & 0.41 & 2.50 & \multicolumn{2}{|c|}{-} & 1.60 & 2.40 & - \\
\hline \multicolumn{9}{|c|}{ Table 9 Comparison of mean concentrations $\left(\mathrm{ng} \mathrm{m}^{-3}\right)$ of total PAHs with other studies } \\
\hline $\begin{array}{l}\text { Total } \\
\text { PAHs } \\
\end{array}$ & \multicolumn{2}{|l|}{ Sampling Site } & \multicolumn{2}{|c|}{ Mean $\left(\mathrm{ng} \mathrm{m}^{-3}\right)$} & \multicolumn{2}{|c|}{ Cities } & \multicolumn{2}{|c|}{ References } \\
\hline$\sum 15$ PAHs & \multicolumn{2}{|c|}{$\begin{array}{l}\text { Residential area (near } \\
\text { industrial area) }\end{array}$} & \multicolumn{2}{|l|}{2.37} & \multicolumn{2}{|c|}{$\begin{array}{l}\text { Turkey. İstanbul. } \\
\text { Tuzla }\end{array}$} & \multicolumn{2}{|l|}{ This study } \\
\hline$\sum 13$ PAHs & \multicolumn{2}{|c|}{ Near coal reserve } & \multicolumn{2}{|c|}{$0.051-1.791$} & \multicolumn{2}{|c|}{ Brazil. Candiota } & \multicolumn{2}{|c|}{$\begin{array}{l}\text { Dallarosa et al. } \\
(2005)\end{array}$} \\
\hline$\sum 13$ PAHs & \multicolumn{2}{|c|}{ Suburban area. traffic } & \multicolumn{2}{|l|}{2.39} & \multicolumn{2}{|c|}{ Japan. Hiroshima } & \multicolumn{2}{|c|}{$\begin{array}{l}\text { Wai Fon et al. } \\
(2007)\end{array}$} \\
\hline$\sum 16$ PAHs & \multicolumn{2}{|l|}{ Urban area } & \multicolumn{2}{|c|}{$28.53-362.15$} & \multicolumn{2}{|c|}{ China. Beijing } & \multicolumn{2}{|c|}{$\begin{array}{l}\text { Bin Liu L, et al. } \\
\text { (2007) }\end{array}$} \\
\hline$\sum 15 \mathrm{PAHs}$ & \multicolumn{2}{|l|}{ Urban area } & \multicolumn{2}{|l|}{86.0} & \multicolumn{2}{|c|}{ China. Nanjing } & \multicolumn{2}{|c|}{$\begin{array}{l}\text { Wang. et al. } \\
(2006)\end{array}$} \\
\hline$\sum 16$ PAHs & \multicolumn{2}{|c|}{ Traffic congestion } & \multicolumn{2}{|c|}{$84.63 \pm 46.66$} & $\begin{array}{l}\text { Turk } \\
\text { Gözt }\end{array}$ & İstanbul. & $\begin{array}{l}\text { Alp and H } \\
\text { (2008) }\end{array}$ & edar \\
\hline$\sum 16$ PAHs & $\begin{array}{l}\text { Local microm } \\
\text { area }\end{array}$ & ological & 26.7 & & Shen & n. China & Liu et al. & \\
\hline $\begin{array}{l}\sum 16 \text { PAHs } \\
\text { (three } \\
\text { sites) }\end{array}$ & $\begin{array}{l}\text { Suburban are } \\
\text { Community a } \\
\text { Traffic conge }\end{array}$ & & $\begin{array}{l}2.7-8.4 \\
3.9-9.1 \\
7.6-16.6\end{array}$ & & $\begin{array}{l}\text { Thai } \\
\text { Mai }\end{array}$ & ad. Chiang & $\begin{array}{l}\text { Chantara } \\
\text { Sangchan }\end{array}$ & \\
\hline
\end{tabular}


When the annual mean of total PAHs concentration of the study that is $2.37 \mathrm{ng} \mathrm{m}$ 3 is compared with other studies, it is seen that there is a remarkable similarity with some suburban areas based on the results. (Wai Fon et al. 2007; Chantara and Sangchan 2009). The other studies have higher total PAH concentrations, since they were realized in crowded urban areas or congested traffic (Table 9).

\section{Conclusions}

The annual mean concentration was measured as $2.37 \mathrm{ng} \mathrm{m}-3$ and ranged between $0.05 \mathrm{ng}$ m-3 and $7.4 \mathrm{ng}$ m-3. Higher concentrations of PAHs were seen in cold season. Exceptionally, naphthalene was observed in high concentrations both in hot and cold seasons. Due to the precipitation, high relative humidity and high dust concentration, naphthalene was hold in condensed phase in liquid form whereas it should be in gaseous phase normally. The higher measurements were seen in $\mathrm{BeP}$ concentrations in hot and cold seasons. BeP, $\mathrm{BbF}, \mathrm{BkF}, \mathrm{CPcdP}, \mathrm{Pyr}$ and Nap were the PAHs that frequently observed in high concentrations. The good correlations occurred between 4 and 5 rings compounds. The TEFs found in recommended limit for hot and cold season as $0.04 \mathrm{ng} \mathrm{m-3}$ and 0.49 ng m-3 respectively. Molecular ratios dedicate that diesel emissions and gasoline from the vehicular emissions are the main pollutant source to İstanbul-Tuzla atmosphere.

\section{Acknowlegments}

We thank to Prof. Dr. M. C. BARLA and technicians of the Piri Reis University, for their help during the collection of aerosol samples.

\section{References}

Alp K, Hanedar A (2008) İstanbul atmosferinde polisiklik hidrokarbon (PAH) konsantrasyonu: 2006-2007 peryodu, Kent Yönetimi, İnsan ve Çevre Sorunları'08 Sempozyumu, 02-06 Kasım
2008, İstanbul Büyükşehir BelediyesiİSTAÇ 321-328.

Aslan, O. and Akyürek, Ö. (2018). Spatial Modelling of Air Pollution from PM10 and SO2 concentrations during Winter Season in Marmara Region: 2013-2014. IJEGEO 5(1): 1-16.

Azimi S, Rocher V, Muller M, Moilleron RR, Thevont DR (2005) Sources, distribution and variability of hydrocarbons and metals in atmospheric deposition in an urban area (Paris, France). Science of the Total Environment 337: 223-239.

Bi X, Sheng G, Peng P, Chen, Y, Zang Z, Fu K (2003) Distribution of particulate- and vapor-phase n-alkanes and polyaromatic hydrocompounds in urban atmosphere of Guangzhou, China. Atmospheric Environmoint 37: 289-298.

Bin Liu L, Hashi Y, Liu M, Wei Y, Ming Lin J (2007) Determination of particleassociated polycyclic aromatic Hydrocarbons in urban air of Beijing by GC/MS. Analytical Sciences Vol. 23.

Bourotte C, Forti MC, Taniguchi S, Bicego MC, Lotufo PA (2005) A wintertime study of PAHs in fine and coarse aerosols in Sao Paulo city, Brazil. Atmospheric Environment 39: 3799-3811.

Bull K (2003) Protocol to the 1979 Convention on Long-Range Trans boundary Air Pollution on Persistent Organic Pollutants: The 1998 Agreement for the UNECE Region, Geneva. The

Burak, S., Ünlü, S. and Gazioğlu, C. (2009). Environmental stress created by chemical pollution in the Marmara Sea (Turkey). Asian Journal of Chemistry, Vol. 21(4). 3166-3174.

Handbook of Environmental Chemistry Vol. 3, Part O, Persistent Organic Pollutants. (ed. By H. Fiedler). Springer-Verlag Berlin Heidelberg.

Chantara S, Sanghan W (2009) Sensitive analytical method for particle-bound polycyclic aromatic hydrocarbons: A case study in Chang Mai, Thailand. Science Asia 35: 42-48.

Chester R (1990) Marine Geochemstry. Unwin. Hyman Ltd, London.

Dallarosa JB, Teixeira EC, Pires M, Farchel J (2005) Study of the profile of polycyclic 
hydrocarbons in atmospheric particles (PM10) using multivariate methods. Atmospheric Environment 39: 65876596.

EPA Environment Protect Agency, 2003. Standard Methods, 5030C.

EPA Environment Protect Agency, 2003. Standard Methods, 3620B

Esen F, Taşdemir Y, Vardar N (2008) Atmospheric concentrations of PAHs, their possible sources and gas-to particle partitioning at a residential site of Bursa, Turkey. Atmospheric Research 88: 243255.

Fang GC, Chang K, Lu C, Bai H (2004) Estimation of PAH's dry deposition and BaP Toxic Equivalency Factors (TEFs) study at urban, industry park and rural sampling sites in Central Taiwan, Taichung, Chemosphere 55: 787-796.

Fang GC, Wu YS, Chen MH, Ho TT, Huang SH, Rau JY (2004) Polyaromatic hydrocarbons study in Taichung, Taiwan, during 2002-2003. Atmospheric Environment 38: 3385-3391.

Gaga EO, Tuncel SG (2003) Occurrence and distribution of polycyclic aromatic hydrocarbons in Ankara precipitation. Water, Air, and Soil Pollution: Focus 3:127-134.

Henner P, Schiavion M, Morel JL, Lichtouse E (1997) Polycyclic aromatic hydrocarbon (PAH) occurrence and remediation methods. Analuis Magazine 25:56-59.

Khalli NR, Scheff PA, Holsen TM (1995) PAHs source fingerprints for coke ovens, diesel and gasoline engineers, highway tunnels, and wood combustion emissions. Atmospheric Environment 29:533-542.

Li CK, Kamens RM (1993) The use of polycyclic aromatic hydrocarbons as sources signatures in receptor modeling. Atmospheric Environment 159:185-200.

Liu G, Tong Y, Luong JH, Zhang H, Sun H (2010) A source study of atmospheric polycyclic aromatic hydrocarbons in Shenzhen, South China. Environ Monit Asses 163:599-606.

Liu Y, Liu L, Lin JM, Tang N, Hayakawa K (2006) Distribution and characterization of polycyclic aromatic hydrocarbon compounds in airborne particulates of
East Asia, China. Particuology 4(6):283296.

Liu X, Zhang G, Jones KC, Li X, Peng X, Qi $S$ (2005) Compositional fractionation of polycyclic aromatic hydrocarbons (PAHs) in moses (hypnum plumaeformea WILS) from the Nanling Mountains, South China. Atmospheric Environment 39:5490-5499.

Liu WX, Dou H, Wei ZC, Chang B, Qiu WX, Liu Y, Tao S (2009) Emission characteristics of polycyclic aromatic hydrocarbons from combustion of different residential coals in North China. Science of the Total Environment 407:1436-1446.

Mastral AM, Callen MS (2000) A review on polycyclic aromatic hydrocarbon (PAH) emissions from energy generation. Environmental Science and Technology 34:3051-3057.

Nispet C, Lagoy P (1992) Toxic equivalency factors (TEFs) for polycyclic aromatic hydrocarbons (PAHs), Regulatory Toxicology and Pharmacology16:290300.

Panther BC, Hooper MA, Tapper NJ (1999) A comparison of air particulate matter and associated polycyclic aromatic hydrocarbons in some tropical and temperate urban environments. Atmospheric Environment 33:4087-4099.

Pekey B, Karakaş D, Ayberk S (2007) Atmospheric deposition of polycyclic aromatic hydro compounds to İzmit Bay, Turkey. Chemosphere 67:537-547.

Sharma H, Jain VK, Khan ZH (2007) Characterization and source identification of polycyclic aromatic hydrocarbons (PAHs) in the urban environment of Delhi, Chemosphere 66:302-310.

Shen Z, Ha Y, Cao J, Tian J, Zhu C, Liu S, Liu P, Wang Y (2010). Characteristics of traffic-related emissions: A case study in roadside ambient air over Xi'an, China, Aerosol Air Quality Researh 10:292-300.

Skarek M, Cupr P, Bartos J, Kohoutek J, Klanova I, Holubek I (2007) A combined approach to the evaluation of organic air pollution- A case study of urban air in Sarejevo and Tuzla (Bosnia and Herzegovina). Science of the Total Environment 384:182-193. 
Sarrazin L, Diana C, Wafo E, PichardLagadec V, Schembri T, Monod J (2007) Determination of polycyclic aromatic hydrocarbons (PAHs) in marine, brackish, and river sediments by HPLC, Following Ultrasonic Extraction 29:1: 6985.

Sisovic A, Belsic A, Sega K, Vadjic V (2008) PAH mass concentrations measured in PM10 particle fraction. Environmental International 34:580-584.

Tan JH, Bi XH, Duan JC, Rahn KA, Sheng GY, Fu JM (2006) Seasonal variation of particulate polycyclic hydrocarbons associated with PM10 in Guangzhou, China. Atmospheric Research 80:250262.

US EPA Environment Protect Agency, 2003. Standard Methods, 5030C.

Wai Fon TY, Noriatsu O, Hiroshi S (2007) Polycyclic aromatic hydrocarbons (PAHs) in the aerosol of Higashi Hiroshima, Japan: Pollution scenario and source identification, Springer Science + Business Media B.V.

Wang G, Huang L, Zhao X, Niu H, Dai, Z (2006) Aliphatic and polycyclic aromatic hydrocarbons of atmospheric aerosols in five locations of Nanjing urban area, China, Atmospheric Research 81:54-66.

WHO, World Health Organization (1989) IARC Monographs (Vol. 46). Lyon. France: International Agency for Research on Cancer 41-155.

WHO Regional Office for Europe (2000) Air quality guidelines. Second edition. Copenhagen, Denmark 